\title{
RECEPTOR-INDEPENDENT SPREAD OF A NEUROTROPIC MURINE CORONAVIRUS MHV-JHMV IN MIXED NEURAL CULTURE
}

\author{
Keiko Nakagaki, Kazuhide Nakagaki, and Fumihiro Taguchi*
}

\section{INTRODUCTION}

Highly neurovirulent mouse hepatitis virus (MHV) JHMV strain multiplies in a variety of brain cells, although the expression of its receptor, carcinoembryonic antigen cell adhesion molecule 1 (CEACAM 1, MHVR), is expressed only in microglia/macrophages in the brain. ${ }^{1}$ The present study was undertaken to clarify the mechanism of extensive JHMV infection of the brain by using neural cells isolated from mice.

\section{MATERIALS AND METHODS}

\subsection{Virus, Neural Cell Culture, and Antibody}

We used two strains of MHV: MHV-JHM cl-2 strain (designated wt JHMV), known to be a neurotropic MHV, and srr7 (soluble receptor resistant mutant), derived from wt JHMV. Srr7 has a single amino acid mutation in the S protein (L1114F). ${ }^{2}$ Primary mixed neural cell cultures were established from the forebrains of 1- to 3-day-old neonate mice as described previously with minor modifications. ${ }^{3}$ Primary antibodies used for immunocytochemistry or flow cytometry (FACS) to identify each cell type were as follows; anti-glial fibrillary acidic protein (GFAP) polyclonal antibody for astrocytes, O4 monoclonal antibodies (MAbs) for oligodendrocytes, and MAP-2 for neuron. Binding of the Griffonia simplicifolia lectin (GS-lectin) was used for microglia/macrophages identification. To detect MHV-specific antigen in cells, mouse anti-MHV MAbs were used. ${ }^{4}$ MAb CC1 to detect MHVR and to block the infection was kindly provided by Kay V. Holmes. 5

\footnotetext{
* Keiko Nakagaki, Fumihiro Taguchi, National Institute of Infectious Diseases, Tokyo 208-0011, Japan Kazuhide Nakagaki, Nippon Veterinary and Animal Science University, Tokyo 180-8602, Japan.
} 


\section{RESULTS}

\subsection{Infection of wt JHMV or srr-7 in Cultured Neural Cells and the Blockade of Initial Infection by Anti-MHVR MAb CC1}

Virus-infected fused cells were sporadically detected in neural cell culture at $8 \mathrm{~h}$ after wt JHMV infection and the infection rapidly spread regardless of cell type. Twenty-four hours postinfection (PI), the majority of cells were included in syncytia and contained viral antigens. Unlike wt JHMV, the viral antigen in srr7-infected cells were detected in only a small proportion of club-like cells at 12 hour, PI, and syncytium formation was not observed as long as 24 hour, PI. The cells infected with srr7 were immunocytochemically identified as microglia/macrophages because of GS-lectin-positivity. Because the virus infection was blocked by the pretreatment of neural cells with $\mathrm{CC} 1$, we concluded that initial infection of those two viruses was mediated by MHVR.

\subsection{Identification of MHVR-positive Cells and of Cells That Bound JHMV by FACS}

Neural cells isolated from the cerebrum of neonatal mice were directly examined for GS-lectin positivity as well as for the presence of MHVR. MHVR-positive cells stained with $\mathrm{CC} 1$ accounted for $2.1 \%$ of the entire neural cell population, while GS-lectinpositive cells were $5.1 \%$. Approximately $78 \%$ of the MHVR-positive cells were revealed to be GS-lectin positive, showing that the major population expressing MHVR were microglia/macrophages. In contrast, $48 \%$ of GS-lectin-positive cells were MHVRpositive, indicating that half of the microglia/macrophages express MHVR and the other half does not. Virus-bound cells accounted for only $1 \%$ of all cells isolated from brain, but more than $83 \%$ of those cells were GS-lectin-positive cells. This suggests that most of the cells bound with JHMV are microglia/ macrophage.

\subsection{Virus Infection in Microglia/Macrophages-Enriched Culture}

In GS-lectin-positive microglia/macrophages-enriched cultures, wt and srr7 similarly induced syncytia in approximately one-half of cultured cells. The finding indicates that both viruses spread and efficiently induce syncytium formation in microglia/macrophage cultures.

\subsection{Effects of CC1 Treatment on Virus Spread from Initially Infected Cells}

The results presented above are suggestive that the wt JHMV spreads from primarily infected microglia/macrophages to MHVR-negative cells in mixed neural cell culture, while srr7 infection is limited to MHVR-positive microglia/macrophages. To see whether this is a case or not, we have examined whether CC1 prevents wt spread from initially infected cells. Neural cells were infected with wt JHMV, and 1 hour later CC1 was added in the culture. There was no substantial difference in the proportion of wt JHM antigen-positive syncytium formation, when cultured in the presence or absence of CC1. This indicates that wt virus spread from initially infected cells is not mediated with MHVR. We have also done a similar experiment using either wt or srr7 in microglia/macrophage-enriched cultures. $\mathrm{CC} 1$ prevented the spread of srr7, while it 


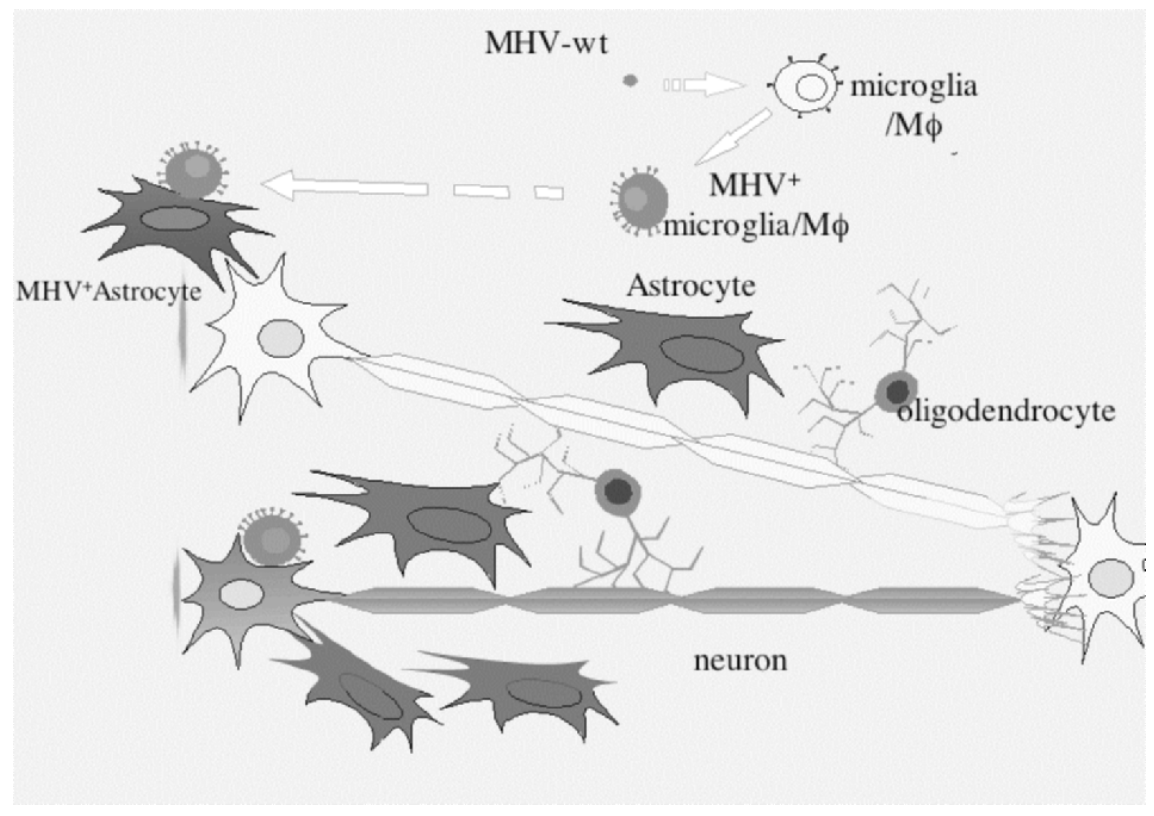

Figure 1. Schematic representation of mechanisms of MHVR-independent infection caused by wt JHMV in neural cells. The virus spreads from initially infected microglia/macrophages $(\mathrm{m} \phi)$ to receptor-negative cells.

failed to prevent the wt virus spread in a similar way observed in srr7 infection, though it reduced wt syncytium formation to a certain extent. These results clearly showed that wt virus spread from initially infected cells to a variety of cells in an MHVR-independent fashion, while srr7 infection was solely MHVR-dependent.

\section{CONCLUSIONS}

The present study indicates that microglia/macrophages are the major population expressing MHVR in the brain, and they are the initial targets for MHV. Wt JHMV spreads from initially infected microglia/macrophages, which is MHVR-dependent manner, to a variety of cells such as astrocytes, oligodendrocytes or neuron in an MHVR-independent fashion as postulated by Gallagher and Buchmeier ${ }^{6}$ (Fig. 1).

\section{ACKNOWLEDGMENTS}

We greatly thank Dr. Kay V. Holmes for MAb specific for MHVR (CC1). This work was partly supported by a grant ("Urgent Research on the Diagnosis and Test Techniques for SARS") from the Ministry of Education, Science, Sports and Culture of Japan. 


\section{REFERENCES}

1. Gallagher, T. M., and Buchmeier, M. J., 2001, Coronavirus spike proteins in viral entry and pathogenesis, Virology 279:371-374.

2. Hirayama, M., Silberberg, D. H., Lisak, R. P., and Pleasure, D., 1983, Long-term culture of oligodendrocytes isolated from rat corps callosum by percoll density gradient. Lysis by polyclonal antigalactocerebroside serum, J. Neuropathol. Exp. Neurol. 42:16-28.

3. Kubo, H., Takase, Y-S., and Taguchi, F., 1993, Neutralization and fusion inhibition activities of monoclonal antibodies specific for S1 subunit of the spike protein of neurovirulent murine coronavirus HMV cl-2 variant, J. Gen. Virol. 74:1421-1425.

4. Ramarkrishna, C., Bergmann, C. C., Holmes, K. V., and Stohlman, S., 2004, Expression of the mouse hepatitis virus receptor by central nervous system microglia, J. Virol. 78:7828-7832.

5. Saeki, K., Ohtsuka, N., and Taguchi, F., 1997, Identification of spike protein residues of murine coronavirus responsible for receptor-binding activity by use of soluble receptor-resistant mutants, J. Virol.71:9024-9031.

6. Williams, R. K., Jiang, G. S., Snyder, S. W., Frana, M. F., and Holmes, K. V., 1990, Purification of the 110-kilodalton glycoprotein receptor for mouse hepatitis virus (MHV)-A59 from mouse liver and identification of a nonfunctional, homologous protein in MHV-resistant SJL/J mice, J. Virol. 64:3817-3823. 